\title{
Momentum transfer for momentum transfer-free which-path experiments
}

\author{
Aurélien Drezet* Andreas Hohenau, and Joachim R. Krenn \\ Institute of Physics, Karl-Franzens University, Universitätsplatz 5, A-8010 Graz, Austria
}

(Dated: July 18, 2018)

\begin{abstract}
We analyze the origin of interference disappearance in which-path double aperture experiments. We show that we can unambiguously define an observable momentum transfer between the quantum particle and the path detector and we prove in particular that the so called "momentum transfer free" experiments can be in fact logically interpreted in term of momentum transfer.
\end{abstract}

PACS numbers: 03. 65. Ta, 32. 80. Lg, 07. 79. Fc

\section{INTRODUCTION}

Bohr's principle of complementarity constitutes the hallmark and one of the most intriguing features of quantum mechanics. On the basis of this principle [1, 2] it is indeed universally accepted that any devices capable of determining the path taken by a particle trough a Young like double-aperture must destroy the interference. The justification usually presented is based on Heisenberg's uncertainty principle [3, 4] and involves an irremediable exchange of momentum between the system considered and the measuring apparatus. Over the last decades the primacy of such recoil arguments has been however contested in favor of a more general decoherence mechanism considering the entanglement of the observed system with its environment 5]. In particular Scully et al. emphasized [ 6$]$ that an atom, after emitting a long wavelength photon in a "micromaser-cavity", located close to one of the two pinholes, can generate a recordable whichpath information without transfer of significant momentum. The conclusions of [ $[$ ] seem actually verified in experiments using entanglement with an internal degree of freedom to label the path with either neutrons 7], photons $8,[36]$, or atoms 10, 11].

This stirred-up considerable controversy and a debate 12, 13, 14, 15, 16, 17, 18, 19, 20, 21, 22] on the genuine meaning of momentum transfer in which-path experiments. The paradox comes from the fact that any far-field interference observed with a Young's like double aperture experiment is a direct map of the transverse momentum distribution of the diffracted particle in the aperture plane. Any processes able to erase the interference should be then interpretable in term of a perturbation done on this momentum distribution. Such proposition was formally done 12, 13, 14 but the answer is far from being universally accepted [15, 16]. We found two reasons for that: Firstly, the momentum distribution of the particle in the single-aperture experiment is not affected by the detector (this intuitively implies a momentum transfer equal to zero [ 6$]$ ). Secondly, the momentum transfer defined in 12, 13] is in general "hidden" in the

*Electronic address: aurelien.drezet@uni-graz.at sense that it is not always connected with an experimentally recordable momentum (like the photon momentum in the Feynman light microscope [23]).

In this article we revive this controversy by proposing a consistent definition of momentum transfer based only on the concept of quantum observables. We study the generality of the recoil mechanism and show in particular why the scheme proposed in [6] is not recoil-free. We analyze in this context the roles played by both Heisenberg's relation and entanglement.

\section{TWO FUNDAMENTAL EXAMPLES OF WHICH PATH EXPERIMENTS}

We consider at first the scheme suggested in [6] and sketched in Fig. 1. In the absence of any photon emission (see Fig. 1 (a)) the atomic state immediately behind the holes evolves into a sum of two diffracted waves: $\Psi_{I}(\mathbf{r})=\Psi_{A}(\mathbf{r})+\Psi_{B}(\mathbf{r})$, where $\Psi_{A, B}$ are single aperture wave function reducing to two narrow peaks located at $\mathbf{r}_{A, B}= \pm d / 2 \hat{\mathbf{x}}$ in the aperture plane ( $d$ being the distance between the holes). The which-path detectors consist of two micromaser empty cavities, one placed at each aperture $A$ and $B$ of a Young interferometer. Before passing through the double-aperture, an atom initially in a longlived Rydberg exited state $|e\rangle$, will radiate a photon in one or the other cavity and will finish its journey in the ground state $|g\rangle$ (see Fig. 1 (b)). The joint wave function atom-photon is actually an entangled state which carries the position ambiguity of the molecule over to an ambiguity of the photon state:

$$
\left|\Psi_{J}\right\rangle \simeq \int d^{3} \mathbf{r}\left[\Psi_{A}(\mathbf{r})\left|\gamma_{A}\right\rangle+\Psi_{B}(\mathbf{r})\left|\gamma_{B}\right\rangle\right]|\mathbf{r}\rangle
$$

Here $\left|\gamma_{A, B}\right\rangle$ are single photon states well localized in one or the other cavity. Such condition can create a distinguishability since the photon states are orthogonal e. g. $\left\langle\gamma_{B} \mid \gamma_{A}\right\rangle=0$. In the far-field of the apertures the intensity collected on the screen is proportional to $G^{(1)}(\mathbf{r})=\operatorname{Tr}[\hat{\rho}|\mathbf{r}\rangle\langle\mathbf{r}|]$ (with the density operator $\left.\hat{\rho}=\left|\Psi_{J}\right\rangle\left\langle\Psi_{J}\right|\right)$ and we deduce

$$
G_{S}^{(1)}(\mathbf{r}, t) \propto 1+\mathcal{V} \cos \left[p_{x} d / \hbar+\phi\right],
$$

where the visibility $\mathcal{V}=\left|\left\langle\gamma_{B} \mid \gamma_{A}\right\rangle\right|$ and phase shift $\phi=$ $\arg \left(\left\langle\gamma_{B} \mid \gamma_{A}\right\rangle\right)$ are identically equal to zero and where 

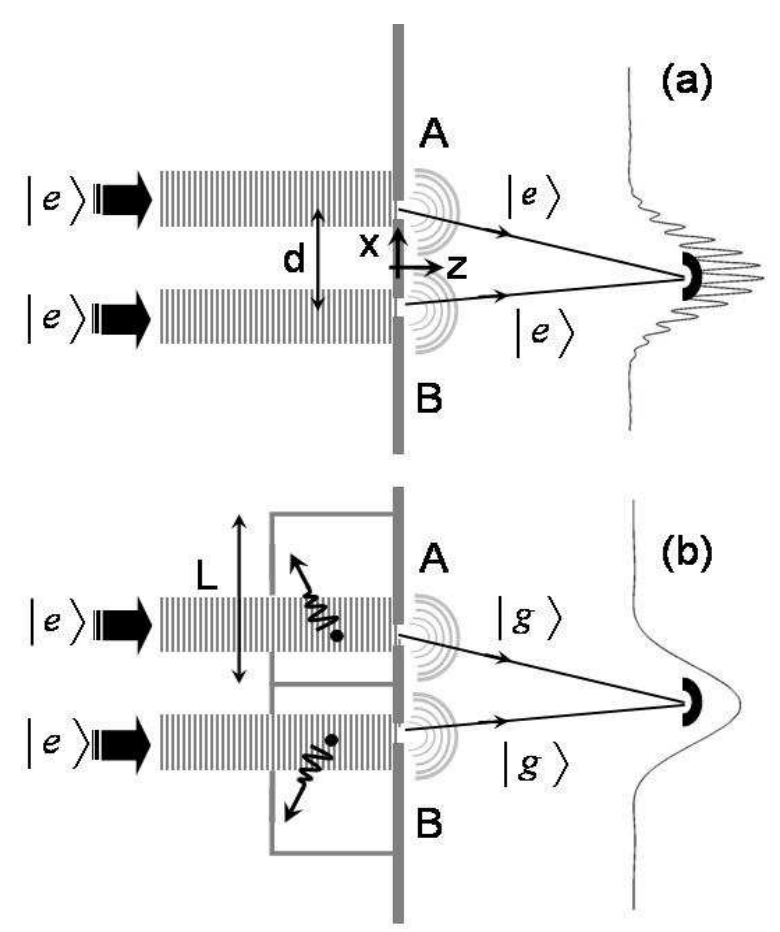

FIG. 1: Sketch of Young's double-hole experiment for one atom as discussed in ref. [6] and in the present article. The separation between the pinholes $A$ and $B$ is $d$ and the aperture screen is located in the plane $x=0$. (a) Without which-path detectors we observe interference fringes with high contrast. (b) Oppositely, if the two micromaser cavities of length $L \gg d$ are introduced the atom radiates a photon giving the which path information. As a consequence of Bohr's complementarity the fringes must disappear.

$p_{x}=\hat{\mathbf{x}} \cdot \hat{\mathbf{r}} h / \lambda_{d B}$ is the transverse atomic momentum for a particle with de Broglie wavelength $\lambda_{d B}$. There is clearly a one to one relation between $G_{S}^{(1)}(\mathbf{r}, t)$ and the momentum distribution $P\left(p_{x}\right)$ of the atom in the aperture plane and we will accept the generality of this relationship in the following.

It is remarkable however that equations similar to Eqs. 12 can be written in the case of an atom emitting spontaneously a photon while it is still in the vicinity of the double-pinhole. Supposing the non-relativistic approximation and that the de-excitation occurs sufficiently fast behind the pinhole one obtains in this Heisenberg like which path experiment $\left\langle\gamma_{B} \mid \gamma_{A}\right\rangle=F_{0}\left(k_{\gamma} d\right)$ [24, 25, 26] which tends to zero for photon wavelength $\lambda_{\gamma}=2 \pi / k_{\gamma}$ much smaller than $d$. This is indeed in agreement with Heisenberg's back-action argument.

We justify the recoil mechanism at work during the spontaneous emission process by expanding the photon states in the wave-vector/polarization-vector basis:

$$
\left|\gamma_{A, B}\right\rangle=\sum_{\mathbf{k}, \epsilon} \gamma_{\mathbf{k}, \epsilon}^{A, B}|\mathbf{k}, \epsilon\rangle=\sum_{\mathbf{k}, \epsilon} \gamma_{\mathbf{k}, \epsilon}^{(0)} e^{-i \mathbf{k} \cdot \mathbf{r}_{A, B}}|\mathbf{k}, \epsilon\rangle
$$

where $\gamma_{\mathbf{k}, \epsilon}^{(0)}$ is the photon amplitude for a hole centered at $x=y=z=0$ [27]. In this context Eq. 1 becomes

$$
\left|\Psi_{J}\right\rangle \simeq \int d^{3} \mathbf{r} \sum_{\mathbf{k}, \epsilon}\left[\Psi_{A}(\mathbf{r}, t) \gamma_{\mathbf{k}, \epsilon}^{A}+\Psi_{B}(\mathbf{r}, t) \gamma_{\mathbf{k}, \epsilon}^{B}\right]|\mathbf{k}, \epsilon, \mathbf{r}\rangle
$$

and the atomic intensity recorded on the screen is consequently

$$
G_{H}^{(1)}(\mathbf{r}) \propto P_{H}\left(p_{x}\right)=\sum_{\mathbf{k}, \epsilon} P\left(p_{x}, \mathbf{k}, \epsilon\right)
$$

with

$P\left(p_{x}, \mathbf{k}, \epsilon\right)=\left|\tilde{\Psi}^{0}\left(p_{x}+\hbar k_{x}\right)\right|^{2} \cdot\left|\gamma_{\mathbf{k}, \epsilon}^{(0)}\right|^{2}\left[1+\cos \left(p_{x} d / \hbar+\mathbf{k} \cdot \mathbf{d}\right)\right]$,

and where $\tilde{\Psi}^{0}\left(p_{x}\right)$ is the Fourier transform of the single aperture wave function $\Psi^{0}(\mathbf{r})$ centered at the origin. The tiny broadening of the single aperture wave function is not fundamental and we can make the approximation $\tilde{\Psi}^{0}\left(p_{x}+\hbar k_{x}\right) \simeq \tilde{\Psi}^{0}\left(p_{x}\right)$ valid for narrow apertures.

We can see that the momentum transferred to the photon by the atom or molecule affects the coherence of the recorded signal. This is clearly visible from the fact that $G_{H}^{(1)}$ is a sum of patterns with unit visibility shifted by an amount $\phi_{\mathbf{k}}=\mathbf{k} \cdot \mathbf{d}=k_{x} d$. Each individual pattern is unable in itself to erase the fringes but the sum of all these patterns can do it. Indeed from the values of the coefficients $\left|\gamma_{\mathbf{k}, \epsilon}^{(0)}\right|^{2}[27,28,29]$ we deduce that the uncertainty $\delta k_{x}$ on the x-component of the photon wave vector is sufficient to account for the disappearance of fringes (this is effectively true because $\delta k_{x} \sim 2 \pi / \lambda_{\gamma} \gg 2 \pi / d$ and $\left.\delta \phi_{\mathbf{k}} \gg 1\right)$. The detailed calculation is straightforward. The coefficients

$$
\left|\gamma_{\mathbf{k}, \epsilon}^{(0)}\right|^{2} \propto \frac{\left|\boldsymbol{\mu}_{g e} \cdot \epsilon\right|^{2}}{\left(\omega-\omega_{\gamma}\right)^{2}+\Gamma^{2} / 4}
$$

are obtained for a two level atom with life time $\Gamma^{-1}$ and transition energy $\hbar \omega_{\gamma}$. After summing on photon polarization $\epsilon$ one deduces:

$$
\sum_{\epsilon}\left|\gamma_{\mathbf{k}, \epsilon}^{(0)}\right|^{2} \propto \frac{\left(\left|\boldsymbol{\mu}_{g e}\right|^{2}-\left|\boldsymbol{\mu}_{g e} \cdot \mathbf{k}\right|^{2} / k^{2}\right)}{\left(\omega-\omega_{\gamma}\right)^{2}+\Gamma^{2} / 4}
$$

A numerical calculation of Eq. 5 requires evaluation of the sum $\mathcal{F}\left(k_{\gamma} d\right)=\sum_{\mathbf{k}} \frac{\left(\left|\boldsymbol{\mu}_{g e}\right|^{2}-\left|\boldsymbol{\mu}_{g e} \cdot \mathbf{k}\right|^{2} / k^{2}\right)}{\left(\omega-\omega_{\gamma}\right)^{2}+\Gamma^{2} / 4} e^{i \mathbf{k} \cdot \mathbf{d}}$. One obtains 27]

$$
\mathcal{F}\left(k_{\gamma} d\right)=\left[\sum_{\mathbf{k}} \frac{\left(\left|\boldsymbol{\mu}_{g e}\right|^{2}-\left|\boldsymbol{\mu}_{g e} \cdot \mathbf{k}\right|^{2} / k^{2}\right)}{\left(\omega-\omega_{\gamma}\right)^{2}+\Gamma^{2} / 4}\right] F_{0}\left(k_{\gamma} d\right)
$$

and

$$
G_{H}^{(1)}(\mathbf{r}, t) \propto 1+F_{0}\left(k_{\gamma} d\right) \cos \left[p_{x} d / \hbar\right],
$$


where $F_{0}\left(k_{\gamma} d\right)$ is calculated in $30,31,32,33$. In particular for an isotropic distribution of transition dipoles we have

$$
\left\langle F_{0}\left(k_{\gamma} d\right)\right\rangle=\sin \left(k_{\gamma} d\right) /\left(k_{\gamma} d\right) e^{-\Gamma d /(2 c)} \simeq \sin \left(k_{\gamma} d\right) /\left(k_{\gamma} d\right)
$$

which indeed tends to zero for $\lambda_{\gamma} \ll d$. This result is in agreement with the complementarity principle since the fringe visibility depends on the amount of information that is in principle available to an outside observer. It is consistent with Heisenberg's back-action argument, since those photons that provide a better path-information also impart a stronger recoil. And finally it is derived by decoherence theory, which means it is based on the entanglement between the atom and the emitted photon. Equivalently we can write

$$
P_{H}\left(p_{x}\right)=\sum_{\mathbf{k}, \epsilon}\left|\gamma_{\mathbf{k}, \epsilon}^{(0)}\right|^{2} P_{0}\left(p_{x}+\hbar k_{x}\right)
$$

with $P_{0}\left(p_{x}\right)=\left|\tilde{\Psi}_{I}\left(p_{x}\right)\right|^{2}=\left|\tilde{\Psi}_{A}\left(p_{x}\right)+\tilde{\Psi}_{B}\left(p_{x}\right)\right|^{2}$. This is equivalent to Eq. [5 and shows that we can define a momentum transfer having the effect of a correlation function. The problem can be then analyzed semiclassically only by using intuitive arguments based on Heisenberg's relation.

At this point it is relevant to repeat that the transfer of momentum doesn't disturb the single aperture pattern which is not broadened significantly! This is a central issue here. Indeed in both the Heisenberg and the Scully et $a l$. example the spatial wave functions $\Psi_{A, B}(\mathbf{r}, t)$ associated with the center of mass are not affected since they factorize from the photon states. However our treatment of the Heisenberg which-path experiment considers explicitly the momentum transfer. This prompts the question as to whether there is or there is not a recoil mechanism in the experiment proposed by Scully et al.

One should now logically observe that the momentum analysis of Heisenberg's experiment can be extended to the proposal made in [6]. The photon states in the micromaser can indeed be expanded like in Eq. 3 but with different coefficients $\gamma_{\mathbf{k}, \epsilon}^{A, B}$ taking into account the specifical structure of the photon field confined by the cavity walls. In the micromaser setup we consider the electric field being oriented along $x$ and ideally constant inside of the cavity of width $L[6,19]$. In order to prohibit overlap between the two cavities we suppose additionally that they are centered on $x= \pm L / 2$. After straightforward calculations we deduce consequently

$$
\gamma_{\mathbf{k}, \epsilon}^{A, B} \propto \operatorname{sinc}\left(\frac{k_{x} L}{2}\right) e^{ \pm i k_{x} L / 2}=\gamma_{k_{x}}^{(0)} e^{ \pm i k_{x} L / 2}
$$

and we write $G_{S}^{(1)}(\mathbf{r}) \propto P_{S}\left(p_{x}\right)$ with

$$
P_{S}\left(p_{x}\right)=\sum_{k_{x}}\left|\gamma_{k_{x}}^{(0)}\right|^{2}\left[1+\cos \left(p_{x} d / \hbar+k_{x} L\right)\right]\left|\tilde{\Psi}_{0}\left(p_{x}\right)\right|^{2} .
$$

Again we have a sum of oscillation terms with unit visibility like in Eq. 5 but now with a phase shift $\phi_{\mathbf{k}}=k_{x} L$ instead of $k_{x} d$. Considering this difference we deduce that in the Scully et al. scheme one only needs a variation of the wave vector in the interval $\delta k_{x} \sim 2 \pi / L \ll 2 \pi / d$ in order to destroy the fringes. Such variation is made possible because the typical dispersion $\delta k_{x}$ in the Fourier space of the photon is $\sim 2 \pi / L$. The result can be rigorously proven by using the Wiener-Khintchine theorem. Indeed the total visibility of $G_{S}^{(1)}(\mathbf{r})$ is equivalently defined by $\mathcal{V}=\left.\left|\int\right| \gamma_{k_{x}}^{0}\right|^{2} e^{i k_{x} L} d k_{x} \mid$. From Wiener-Khintchine's theorem we deduce $\int\left|\gamma_{k_{x}}^{0}\right|^{2} e^{i k_{x} L} d k_{x}=\int d x f(x+L) f^{*}(x)=$ $0=\mathcal{V}$ (autocorrelation function) where $f(x)=\mathcal{F}^{-1}\left[\gamma_{k_{x}}^{0}\right]$ is the unit rectangular function of width $L$ centered on the origin $x=0$.

It is then not true to say that the photon momentum is not involved in a recoil mechanism since it is only by summing all the oscillating contribution in Eq. 14 that we can account for the decoherence effect.

The comparison becomes more evident if we work in the limit of very narrow apertures neglecting the modification of the single hole diffraction pattern:

$$
P_{S}\left(p_{x}\right) \simeq \sum_{\mathbf{k}, \epsilon}\left|\gamma_{k_{x}}^{(0)}\right|^{2} P_{0}\left(p_{x}+\hbar k_{x} L / d\right) .
$$

The approximation $\left|\tilde{\Psi}_{0}\left(p_{x}+\hbar k_{x} L / d\right)\right|^{2} \simeq\left|\tilde{\Psi}_{0}\left(p_{x}\right)\right|^{2}$ is here justified since $\delta k_{x} L / d \sim 1 / L \cdot L / d=1 / d$ and since we work in the limit $\delta p_{x}^{\text {single hole }} \simeq h / a \gg h / d$ for the single hole of width $a$. Like in Eq. 12 we find a correlation function but here the result is not intuitive because we need an effective momentum transfer

$$
\hbar k_{x} L / d=\hbar \phi_{\mathbf{k}, \epsilon} / d
$$

instead of $\hbar k_{x}$. Since $\hbar \phi_{\mathbf{k}, \epsilon} / d \gg \hbar k_{x}$ this proves that the momentum transferred is much higher that the intuitive and semiclassical expectation $\sim h / L$. This analysis however does not constitute a disproof but actually a confirmation of Heisenberg's mechanism in term of recoil transfer because the effective momentum is typically $\sim h / d$. Both the mathematical treatment leading to Eqs. 12 and 15 are based on a purely quantum analysis of momentum transfer. In the case of Eq. 12 the calculations confirm the intuitive semiclassical reasoning. However in the case of Eq. 15 only a quantum treatment can justify the result when the semiclassical analogy fails.

\section{DISCUSSION: WHICH-PATH INFORMATION AND QUANTUM ERASER}

\section{A. Which-path information and momentum transfer}

The precedent result which can seem rather surprising is in fact very general and not limited to the particular examples of Eqs. 12,15. Consider the general 
which-path state given by Eq. 1 but where the detector states are not necessarily associated with photons and can be expanded in a arbitrary orthonormal basis $|\xi\rangle$ as $\left|\gamma_{A, B}\right\rangle=\sum_{\xi} \gamma_{\xi}^{A, B}|\xi\rangle$. A analysis similar to the previous one leads to

$$
\begin{array}{r}
P\left(p_{x}\right)=\sum_{\xi}\left(\left|\gamma_{\xi}^{A}\right|^{2}+\left|\gamma_{\xi}^{B}\right|^{2}\right)\left[1+\mathcal{V}_{\xi}\right. \\
\left.\cdot \cos \left(\frac{p_{x} d}{\hbar}+\phi_{\xi}\right)\right]\left|\tilde{\Psi}_{0}\left(p_{x}\right)\right|^{2}
\end{array}
$$

where $\phi_{\xi}=\arg \gamma_{\xi}^{A}-\arg \gamma_{\xi}^{B}$ and where $\mathcal{V}_{\xi}$ is the fringes visibility given by

$$
\mathcal{V}_{\xi}=\frac{2\left|\gamma_{\xi}^{A}\right| \cdot\left|\gamma_{\xi}^{B}\right|}{\left(\left|\gamma_{\xi}^{A}\right|^{2}+\left|\gamma_{\xi}^{B}\right|^{2}\right)} .
$$

From the discussion of the Scully et al. and Heisenberg like examples we conclude that if (i) $\mathcal{V}_{\xi}$ equals unity for those terms of the sum Eq. 17 for which $\left|\gamma_{\xi}^{A}\right|^{2}+\left|\gamma_{\xi}^{B}\right|^{2}$ has significant values, and if (ii) in the mean time the phase $\phi_{\xi}$ changes significantly in an interval $\delta \phi_{\xi} \sim 1$ then decoherence will occur and a momentum transfer can be invoked in the basis $\xi$. To be more precise and still in analogy with Eqs. 12,15 we can write in the narrow apertures limit

$$
\begin{array}{r}
P\left(p_{x}\right) \simeq \sum_{\xi}\left(\left|\gamma_{\xi}^{A}\right|^{2}+\left|\gamma_{\xi}^{B}\right|^{2}\right)\left[\left(1-\mathcal{V}_{\xi}\right)\right. \\
\left.\cdot\left|\tilde{\Psi}_{0}\left(p_{x}\right)\right|^{2}+\mathcal{V}_{\xi} P_{0}\left(p_{x}+\hbar \phi_{\xi} / d\right)\right]
\end{array}
$$

because we suppose $\tilde{\Psi}_{0}\left(p_{x}+\hbar \phi_{\xi} / d\right) \simeq \tilde{\Psi}_{0}\left(p_{x}\right)$. The regime $\mathcal{V}_{\xi}=1$ was the one considered in both Eqs. 12 and 15. It leads to a simple interpretation in terms of a correlation function with a momentum transfer

$$
p_{\xi}=\hbar \phi_{\xi} / d
$$

$\phi_{\xi}$ and then $p_{\xi}$ are clearly experimentally defined by recording the elementary interference pattern corresponding to $\xi$. In this context

$$
\delta p_{\xi} \cdot d \simeq \hbar \delta \phi_{\xi} \simeq \hbar
$$

plays the role of Heisenberg's relation. Yet in general $\mathcal{V}_{\xi} \neq 1$ and this means that there are some terms in Eq. 19 which are proportional to $1-\mathcal{V}_{\xi}$ and which cannot be interpreted in terms of correlation functions and momentum transfers in the basis $\xi$. At the extreme $\mathcal{V}_{\xi}=0$ and the momentum transfer discussion is completely irrelevant in the basis $\xi$.

However it is not difficult using the arbitrariness in the basis choice to find a representation of the problem in which $V_{\xi}=1$ and in which momentum transfer is clearly defined. This is true assuming the ideal which-path experiment

$$
\left\langle\gamma_{A} \mid \gamma_{B}\right\rangle=0
$$

Indeed this orthogonality relation means that we can use the two states $\left|\gamma_{A, B}\right\rangle$ as a relevant basis for analyzing the problem. In this basis $V_{A, B}=0$. However the equivalent choice

$$
\left|\gamma_{ \pm}\right\rangle=\left(\left|\gamma_{B}\right\rangle \pm\left|\gamma_{A}\right\rangle\right) / \sqrt{2}
$$

is completely pertinent too. In such basis $V_{ \pm}=1$ and Eq. 20 leads to

$$
p_{+}=0, \quad p_{-}=\hbar \phi_{-} / d=h / 2 d .
$$

The momentum $p_{-}$accounts for the loss of coherence in the which-path experiment and we can indeed write

$$
P\left(p_{x}\right)=\sum_{ \pm} P_{0}\left(p_{x}+\hbar \phi_{ \pm} / d\right) .
$$

This means that we can always interpret an ideal which path experiment in terms of momentum transfer and correlation function in at least one basis $\xi$.

\section{B. Erasing knowledge with momentum}

The precedent discussion focussed on the concepts of momentum transfer and which-path information in quantum mechanics. However, we must add here a few further remarks concerning the quantum eraser experiment since the existence of a momentum transfer seems to be in contradiction with the spirit of this experiment. Quantum erasure which was initially proposed in 6, 34] and experimentally realized in [8, 35, 36, 37, 38] has been recently discussed in, e. g., [39, 40]. Such experiments are usually thought as "a way around the uncertainty principle" 6, 29, 40] and one says that in classical physics "this question would never come up" 6]. In order to clarify this point one has first to remind that the philosophy of the quantum eraser proposal is based on the possibility to rewrite an entangled state like

$$
\Psi_{A}(\mathbf{r}, t)\left|\gamma_{A}\right\rangle+\Psi_{B}(\mathbf{r}, t)\left|\gamma_{B}\right\rangle
$$

in the equivalent form

$$
\Psi_{+}(\mathbf{r}, t)\left|\gamma_{+}\right\rangle+\Psi_{+}(\mathbf{r}, t)\left|\gamma_{+}\right\rangle
$$

with

$$
\Psi_{ \pm}(\mathbf{r}, t)=\left(\Psi_{A}(\mathbf{r}, t) \pm \Psi_{B}(\mathbf{r}, t)\right) / \sqrt{2}
$$

and where $\left|\gamma_{ \pm}\right\rangle$are defined by Eq. 23 .

Clearly if one is able to project the photon state in the orthogonal basis $\left|\gamma_{ \pm}\right\rangle$one would be able to retrieves interference fringes (or even anti-interference fringes if the projection is made on $\left.\left|\gamma_{-}\right\rangle\right)$. Since this projection can be delayed the atom fringes can be rebuild (using coincidence measurement techniques) even after that the atom reached the screen 38 . From semi-classical physics this behavior is prohibited 6,41$]$. The most famous quantum 


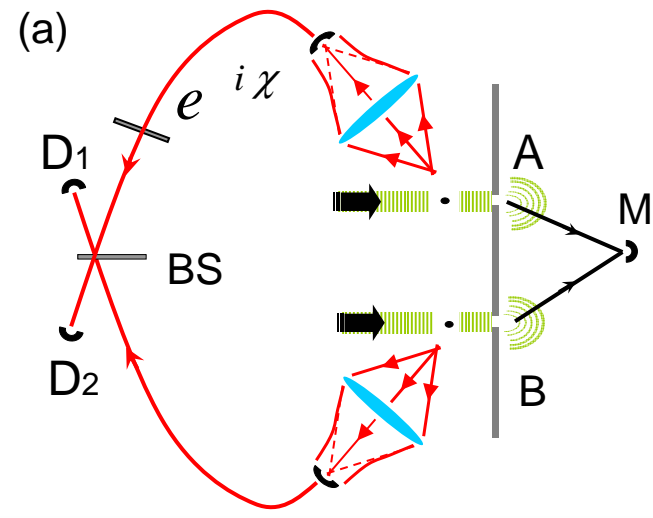

(b)

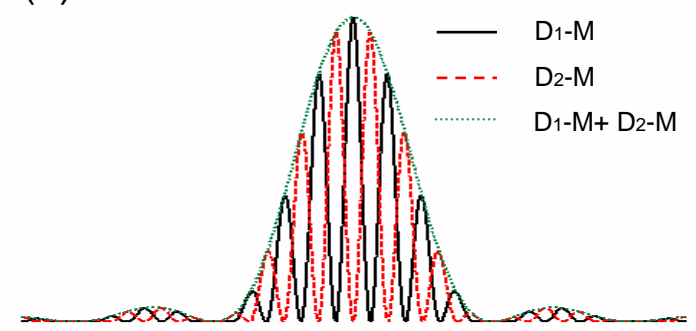

FIG. 2: (color online) a) Quantum eraser experiment using two microscopes for recording a photon spontaneously emitted by the atom close to the aperture A or B. The photon is oriented through optical fibers and a 50-50 beam splitter (BS) before reaching one of the two detectors $D_{1}$ or $D_{2}$. The coincidence measurement atom-photon can be used to rebuild molecular fringes. b) Depending on the phase shift $\chi$ one can observe fringes or anti-fringes. If the correlation $D_{1}$-M gives us the fringes pattern $\left|\Psi_{+}\right|^{2}=\frac{1}{2}\left|\Psi_{A}+\Psi_{B}\right|^{2}$ (black curve) then the correlation $D_{2}$-M gives $\left|\Psi_{-}\right|^{2}=\frac{1}{2}\left|\Psi_{A}-\Psi_{B}\right|^{2}$ (red dashed curve). The sum of both patterns give us the initial distribution $\left|\Psi_{A}\right|^{2}+\left|\Psi_{B}\right|^{2}$ (green dotted envelope).

eraser proposal was given by Scully et al. in [6] and is based on the micromaser two-cavities setup described in section II.

Less know is however the fact that quantum eraser could be in principle realized with the "Heisenberg" setup described in section II and based on spontaneously emitted photons [42]. The principle of this proposal is sketched on Fig. 2a. Detection of single photons emitted in the close vicinity of $\mathrm{A}$ and $\mathrm{B}$ is ensured by microscope objective(s) able to resolve clearly the apertures region. The photon state coming from $\mathrm{A}$ is oriented through an optical fiber to the first entrance of a 50-50 beam splitter (BS). The second photon state follows a similar path to the second entrance of the beam splitter. The recombination of the photon states at the exit outports $D_{1}$ and $D_{2}$ erases the which path information since we have no way to know where the photon was coming from. One could thus retrieve fringes or anti-fringes by changing the phase $\chi$ between the photon paths and by detecting in coincidence the molecule arrival on a screen in $M$ (this experiment is very close to the one realized in [38]). If the Heisenberg experiment described in section II (Eqs. 4-12) was effectively based on (semi) classical momentum kicks associated with the emission of a particule the quantum eraser discussed here could not work! This shows that the semi classical picture is not adapted even in "canonical" experiments like those described by Einstein [1] of Feynman [23].

However, the important word in any quantum eraser experiment is coincidence (i. e., correlation photon-atom). Indeed, if it would be possible to erase the fringes without correlating the detections of the photon and atom then it could be possible to realize faster than light communication [43]. The argument against this possibility is the same as the one used by Bell in the context of Einstein Podolsky Rosen experiments [4]. If we do not compare the data coming from the two exits $D_{1,2}$ with the molecule arrival we must find the initial molecule profile without fringes (see Fig. 2b). This is particulary clear from the fact that we have [6]

$$
\left|\Psi_{+}(\mathbf{r}, t)\right|^{2}+\left|\Psi_{-}(\mathbf{r}, t)\right|^{2}=\left|\Psi_{A}(\mathbf{r}, t)\right|^{2}+\left|\Psi_{B}(\mathbf{r}, t)\right|^{2} .
$$

It must be remarked that this rebuttal of faster than light communication gives us a solution for the paradox concerning the coexistence of momentum transfer with quantum erasure. Indeed, Eq. 29 is tantamount to argumentations based on decoherence theory which say that the entanglement with the environment is responsible for the loss of coherence observed during a which path experiment (we must sum the two quantum eraser patterns $\left|\Psi_{+}(\mathbf{r}, t)\right|^{2}$ and $\left|\Psi_{-}(\mathbf{r}, t)\right|^{2}$ in order to obtain the complete pattern given by $\left|\Psi_{A}(\mathbf{r}, t)\right|^{2}+\left|\Psi_{B}(\mathbf{r}, t)\right|^{2}$, e. g., Eq. 12 or 15).

Now we should remember that the discussion of momentum transfer in section II tells us that we have necessarily to sum all the individual patterns associated with different $|\mathbf{k}\rangle$ in order to obtain the full diffraction pattern without fringes. The present discussion is thus identical to the previous one but is done in the base $\left|\gamma_{ \pm}\right\rangle$instead of $|\mathbf{k}\rangle$. This is completely consistent with section IIIA discussing the observer freedom on the choice of the detector basis $|\xi\rangle$.

In conclusion, since all the argumentation presented in section II is only based on decoherence theory it means that reasonings based on quantum erasers are rigorously equivalent to those based on momentum transfer presented in this article. The quantum eraser corresponds thus precisely to procedures of projection for measuring the momentum transfer. This is visible from Eqs. 23-25 which show indeed that the phase shift between the two quantum eraser patterns $\left|\Psi_{ \pm}(\mathbf{r}, t)\right|^{2}$ corresponds to momentum transfers of $p_{+}=0$ and $p_{-}=h / 2 d$ respectively (e. g, to phase shifts of $\phi_{-}=0$ and $\phi_{+}=\pi$ respectively). It is well known that with a lens focussed on the twoapertures plane one can in principle realize a which path 
experiment. The experimentalist has the choice to position the photon detectors at any distance behind the lens. If the detectors are located in the image plane of the lens one can distinguish the path followed by the atom in the interferometer. However, if the photon detectors are positioned in the back focal plane of the lens (i. e, Fourier plane) then the observer can record the photon momentum distribution in the base $|\mathbf{k}\rangle$. As discussed in section II (i. e., Eq. 6) the joint distribution of probability photon-atom reveals atom fringes with a phase shift $k_{x} d$ which corresponds to a transverse momentum transfer $p_{\mathbf{k}}=\hbar k_{x}$. From this discussion it is thus in principle possible to realize a quantum eraser experiment by correlating photon detection in the back focal plane of the lens with the detection of an atom on the screen. This shows once again that the quantum eraser is indeed connected to the definition of momentum transfer used in this article. The same procedure using a lens could be (in principle) realized with the setup described in [6]. Such an experiment would correspond to a measure of the momentum distribution $p_{\mathbf{k}}=\hbar k_{x} L / d$ (see Eqs. 14-16). However, the presence of the cavities walls constraints in practice such observations with a lens [45]. To realize a quantum eraser it is thus easier to consider the basis $\left|\gamma_{ \pm}\right\rangle$instead of the basis $|\mathbf{k}\rangle$.

The apparent contradiction between the existence of quantum erasers on one hand and the existence of momentum transfer on the other hand results from the (implicit) use of a classical definition of momentum. Once again, with such definition the quantum eraser would not be possible. Here our definition of momentum transfer, e. g. ,

$$
p_{\xi}=\hbar \phi_{\xi} / d
$$

does not have this handicap. This definition is indeed purely quantum and represents the most direct generalization of the usual definition used for the case of Heisenberg's like experiment in Eqs. 4-12. It can be applied to any double-apertures which-path or quantum eraser experiments (like the ones described in []] ).

\section{SUMMARY}

Our discussion of wave-particle duality and of momentum transfer is directly based on the very basis of quantum mechanics which involves only observable quantities associated with states $|\xi\rangle$. This means that the momentum transfer is always in principle experimentally accessible to the observer. This clearly shows the pertinence of our definition in a physical discussion concerning the meaning of momentum transfer. Our analysis show additionally that momentum transfer and quantum eraser are not two independent concepts and that they are in fact two formulations of the same decoherence argumentation applied two double-aperture experiments. Clearly, most of the formalism used here was already known [33]. However, the analysis in term of momentum of experiments like the one proposed in [ $[\underline{6}$. has to our knowledge never been done, consequently missing some fundamental subtleties of the interpretation.
[1] N. Bohr in Albert Einstein philosopher-scientist, edited by P. A. Schilpp, (The library of living philosophers, Evanston, 1949), pp. 200-241.

[2] B. -G. Englert, Phys. Rev. Lett. 77, 2154 (1996).

[3] W. Heisenberg, Z. Phys. 43, 172 (1927).

W. Heisenberg, The physical principles of the quantum theory (University of Chicago Press, Chicago, 1930).

W. Heisenberg, Physics and philosophy (Harper and Brothers, New York, 1958).

[4] W. K. Wootters, and W. H. Zurek, Phys. Rev. D19, 473 (1979).

[5] E. Joos and H. D. Zeh, Z. Phys. B 59, 223 (1985).

[6] M. O. Scully, B. -G. Englert, and H. Walther, Nature (London) 351, 111 (1991).

[7] G. Badurek, H. Rauch and J. Summhammer, Phys. Rev. Lett. 51, 1015 (1983).

[8] S. P. Walborn, M. O. Terra Cunha, S. Padua, and C. H. Monkenet al., Phys. Rev. A65, 033818 (2002).

[36] P. G. Kwiat, A. M. Steinberg and R. Y. Chiao, Phys. Rev. A45, 7729 (1992).

[10] U. Eichmann et al., Phys. Rev. 70, 2359 (1993).

[11] S. Dürr, T. Nonn and G. Rempe, Nature (London) 395, 33 (1998).

[12] P. Storey, S. Tan, M. Collett, and D. Walls, Nature (London) 367, 626 (1994).

[13] H. M. Wiseman and F. Harrison, Nature (London) 377,
$584(1995)$.

[14] H. M. Wiseman et al., Phys. Rev. A. 56, 55 (1997).

[15] B. -G. Englert, M. O. Scully, and H. Walther, Nature (London) 375, 367 (1995).

[16] P. Storey, S. Tan, M. Collett, and D. Walls, Nature (London) 375, 368 (1995).

[17] H. M. Wiseman, Phys. Rev. A58, 1740 (1998).

[18] H. M. Wiseman, Phys. Lett. A. 311, 285 (2003).

[19] B. G. Englert, H. Fearn , M. O. Scully, and H. Walther in Quantum interferometry, edited by F. de Martini, G. Denardo, and A. Zeilinger (World Scientific, Singapor, 1994), pp. 103-119.

[20] P. Storey, S. Tan, M. Collett, and D. Walls in Quantum interferometry, edited by F. de Martini, G. Denardo, and A. Zeilinger (World Scientific, Singapor, 1994), pp. 120129.

[21] B. G. Englert, J. Schwinger, and M. O. Scully in New frontiers in quantum electrodynamics and quantum optics, edited by A. Barut (Plenum, New York, 1990), pp. 513-519.

[22] S. F. Yelin, C. J. Bednar, Chai-Ren Hu, Opt. Commun. 136, 171 (1997).

[23] R. P. Feynman, R. Leighton and M. Sand, The Feynman Lectures on Physics Vol. 3 (Adisson Wesley, Reading, 1965).

[24] T. Pfau et al., S. Spalter, C. Kurtsiefer, C. R. Ekstrom, 
and J. Mlynek,Phys. Rev. Lett. 73, 1223 (1994).

[25] M. S. Chapman, T. D. Hammond, A. Lenef, J. Schmiedmayer, R. A. Rubenstein, E. Smith, D. E. Pritchard, Phys. Rev. Lett. 75, 3783 (1995).

[26] L. Hackermüller et al., Nature (London) 427, 711-714 (2004).

[27] A. Drezet, A. Hohenau, and J. Krenn, Phys. Rev. A73, 013402 (2006).

[28] L. Mandel, J. Optics 1051 (1979).

[29] M. O. Scully and M. S. Zubairy, Quantum optics (Cambridge University Press, Cambridge, 1997).

[30] O. Steuernagel and H. Paul, Phys. Rev. A52, R905 (1995).

[31] K. Rza̧żewski and W. Żakowwicz, J. Phys. B 25, L319 (1992).

[32] C. Brukner and A. Zeilinger, Phil. Trans. R. Soc. London. A360, 1061 (2002).

[33] S. M. Tan and D. F. Walls, Phys. Rev. A47, 4663 (1993).

[34] M. O. Scully and K. Drühl, Phys. Rev. A25, 2208 (1982).

[35] A. G. Zajonc, L. Wang, X. Ou, and L. Mandel, Nature (London) 353, 507 (1991).

[36] P. G. Kwiat, A. M. Steinberg and R. Chiao, Phys. Rev. A45, 7729 (1992); P. G. Kwiat, A. M. Steinberg and R. Chiao, ibid 49, 61 (1994).

[37] T. J. Herzog, P. G. Kwiat, H. Weinfurter, and A. Zeilinger, Phys. Rev. Lett. 75, 3034 (1995).

[38] Y. -H. Kim et al. , Phys. Rev. Lett. 84, 1 (2000).

[39] U. Mohrhoff, Am. J. Phys. 64, 1468 (1996); U. Mohrhoff, ibid 67, 330 (1999).

M. O. Scully and H. Walther, Found. Phys. 28, 5229
(1998).

B. G. Englert, M. O. Scully, and H. Walther, Am. J. Phys. 67, 325 (1999).

[40] Y. Aharonov, M. S. Zubairy, Science 307, 875 (2005).

B. Greene, The fabric of the cosmos (Alfred A. Knopf, New York, 2004).

[41] Consider a model in which the atom is treated by quantum mechanics but where the photon is treated classically as a point particle. Since after its emission the photon will follow a given trajectory we conclude that what ever we could do by acting locally on the photon will not change the fact that atom fringes were already destroyed by the emission process.

[42] T. Pfau, Ch. Kurtsiefer, and J. Mlynek, Quantum Semiclass. Opt. 8, 665 (1996).

[43] E. Jaynes, in foundation of Radiation Theory and Quantum Electrodynamics, edited by A. O. Barut (Plenum, New York, 1980), pp. 37-43.

[44] J. S. Bell, Speakable and Unspeakable in Quantum Mechanics (Cambridge University Press, Cambridge, UK, 1987).

[45] This handicap could be in principle overcome by considering a hypothetical particle crossing the two cavities (in the transversal direction $x$ ) without interacting with the walls. If such particle absorbs the photon one could compare its momentum before and after the interaction and thus rebuild the atom interference pattern (e. g., realize a quantum eraser in the basis $|\mathbf{k}\rangle$ ) by correlating the information obtained with the recording of the atoms. 